\title{
Dyspnea after thyroidectomy induced by intratracheal lymphoma: A case report
}

\author{
CHAOJIE WU ${ }^{1,2}$, DONGZHU DA ${ }^{1,2}$, JING WANG $^{3}$, GAOANG QIAO $^{1,2}$, \\ MIAO ZHENG ${ }^{2}, \mathrm{XU} \mathrm{MING}^{2}$, YONGXIN JIANG ${ }^{2}$ and JUN LIU ${ }^{1,2}$ \\ ${ }^{1}$ Shanghai General Hospital of Nanjing Medical University; ${ }^{2}$ Department of Breast-Thyroid-Vascular Surgery, \\ Shanghai General Hospital; ${ }^{3}$ Shanghai Jiao Tong University School of Medicine Pathology Center, \\ Shanghai 201620, P.R. China
}

Received January 29, 2019; Accepted June 27, 2019

DOI: $10.3892 / \operatorname{mco} .2019 .1896$

\begin{abstract}
The aim of the present study was to report a rare case of dyspnea caused by rapid growth of intratracheal lymphoma in the 2 weeks following thyroid surgery. A 53 -year-old male patient underwent a right thyroidectomy due to tracheal compression by a mass of the right thyroid lobe. However, 2 weeks after the surgery, the patient developed dyspnea, which was managed by tracheotomy. An urgent computed tomography scan revealed that the soft tissue of the upper trachea was significantly thickened compared with prior to surgery, resulting in narrowing of the airway. Postoperative pathology revealed right thyroid lymphoma, and the final diagnosis was intratracheal lymphoma. The intratracheal mass regressed after chemotherapy combined with rituximab. We herein review the diagnostic and treatment process of this case of dyspnea, and analyze the outcome, in order to provide a reference for the timely diagnosis and treatment of patients developing dyspnea following thyroid surgery.
\end{abstract}

\section{Introduction}

Dyspnea is one of the complications of thyroid surgery that may have severe consequences, including death. Common causes of this complication include compression due to formation of a hematoma, tracheal collapse, laryngeal edema and bilateral recurrent laryngeal nerve injury, and they should be taken into consideration to ensure prevention and/or timely treatment of dyspnea $(1,2)$. However, rarer causes may delay diagnosis and lead to a poor outcome. We herein report a case of dyspnea after thyroid surgery, arising from rapid growth of an intratracheal lymphoma. The aim of the present study is to alert physicians to this rare possibility and improve the prognosis for such patients.

Correspondence to: Dr Jun Liu or Dr Yongxin Jiang, Department of Breast-Thyroid-Vascular Surgery, Shanghai General Hospital, 650 Xinsongjiang Road, Shanghai 201620, P.R. China

E-mail: liujun95039@163.com

E-mail: jyxlin@hotmail.com

Key words: dyspnea, post-thyroidectomy, intratracheal lymphoma

\section{Case report}

A 53-year-old male patient was referred to Shanghai General Hospital with a history of a progressively enlarged mass in the right neck for 5 years and dyspnea for 1 month. Physical examination revealed a firm, $10-\mathrm{cm}$ tough mass in the right neck, which did not move on deglutition, without a vascular murmur. B-ultrasonography revealed a hypoechoic nodule, measuring $50 \times 90 \mathrm{~mm}$, in the right lobe of the thyroid gland, with an unclear boundary, inhomogeneous echo, and a dot-like blood flow signal (Fig. 1A). A computed tomography (CT) examination revealed bilateral thyroid enlargement, more prominent on the right side, with narrowing of the trachea and shifting to the left side due to compression (Fig. 1B). No obvious enlargement of the cervical lymph nodes was observed by either B-ultrasonography or CT imaging. Laboratory data revealed normal thyroid function, apart from a high thyroglobulin antibody titer (2,025 IU/ml; normal range 10-115 IU/ml). Given that the right thyroid mass caused dyspnea by compressing the trachea, a right thyroidectomy was performed without preoperative biopsy. During surgery, a solid mass, sized $\sim 10 \mathrm{~cm}$ (Fig. 1C) was identified on the right side of the thyroid gland, of moderate hardness and with a complete capsule. The trachea was compressed and shifted to the left, without tracheomalacia. No obvious adhesions between the tumor and the trachea were observed during surgery. Intraoperative frozen section biopsy revealed marked lymphoid hyperplasia in the right lobe of the thyroid gland (Fig. 2A). Postoperative pathological examination confirmed non-Hodgkin diffuse large B-cell lymphoma of the right thyroid (Fig. 2B). The Ki-67 index was 70\%, indicating rapid tumor proliferation (Fig. 2D). Dyspnea was markedly relieved after surgery, without residual hoarseness, drinking cough, shakiness or emotional changes. Hospitalization was uneventful and the patient was discharged on the third postoperative day.

On the 10th postoperative day, the patient suffered from chest distress and shortness of breath without an obvious inducing factor, and without significant improvement with rest. On the 11th day, the dyspnea was aggravated and the patient was restless, with inspiration difficulty, cyanosis of the lips and inspiratory three depressions sign. Consequently, the patient visited the Emergency Department at Shanghai 

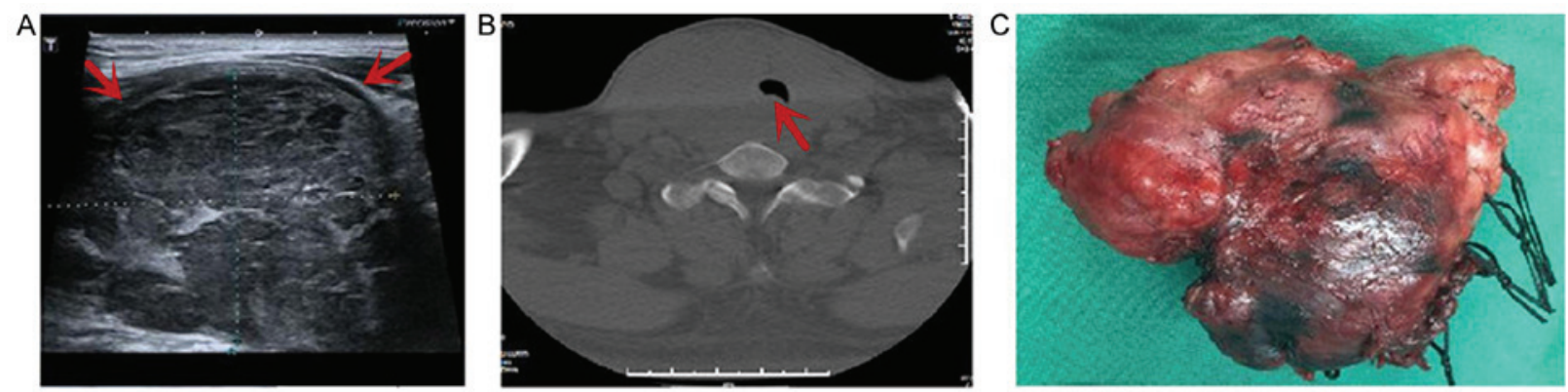

Figure 1. (A) Preoperative B-ultrasound revealed a solid mass in the right lobe of the thyroid gland. (B) Preoperative CT examination revealed compression of the trachea and shift to the left (arrow). (C) Macroscopic appearance of the resected right thyroid mass. CT, computed tomography.
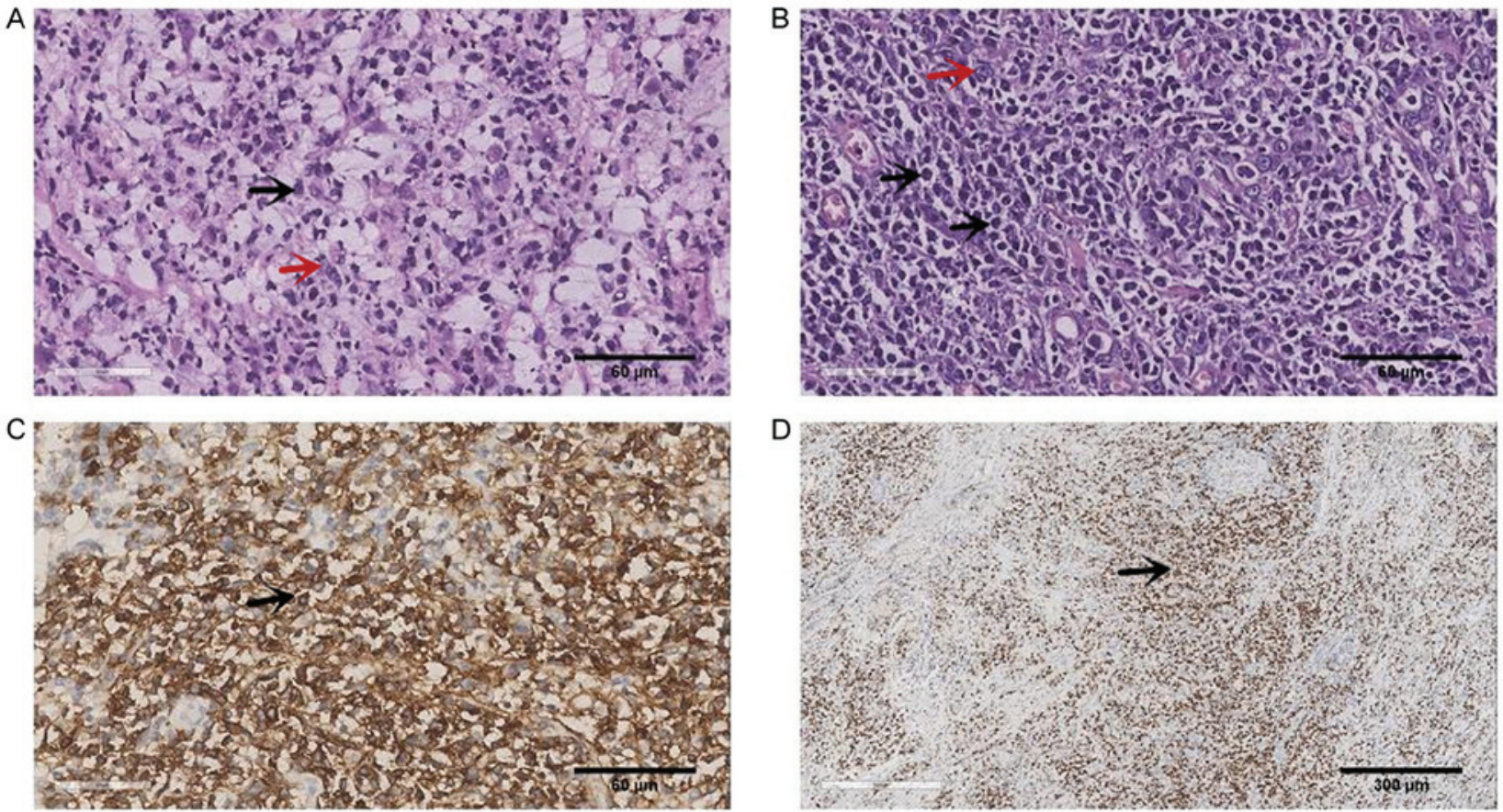

Figure 2. Histopathological characteristics of the right thyroid lymphoma. (A) Intraoperative frozen biopsy examination. (Hematoxylin-eosin staining, magnification, $\mathrm{x} 400$, black arrow $=$ central cell; red arrow $=$ centroblastic cell). (B) Postoperative pathological examination. (Hematoxylin-eosin staining, magnification, $x 400$, black arrow $=$ central cell; red arrow $=$ centroblastic cell). (C) Positive immunohistochemical staining for CD20 (magnification, $x 400$, black arrow $=$ central cell). (D) Positive Ki-67 index $(70 \%)$. (Magnification, $\mathrm{x} 100$, black arrow $=$ central cell).

General Hospital. There was no history of foreign matter aspiration after discharge. B-ultrasound examination revealed no hematoma in the thyroid surgery area, uneven rough echo, and normal blood supply in the residual thyroid gland. The symptoms improved after oxygen inhalation, and a contrast-enhanced CT of the neck was scheduled. On the 15 th postoperative day, during the CT examination, the patient suddenly lost consciousness and went into respiratory arrest, recovering breathing and consciousness only after tracheotomy and assisted ventilation. An urgent neck CT scan revealed that the thyroid gland exhibited widespread enlargement with decreased density. The soft tissue of the subglottic cavity and upper trachea was notably thickened compared with prior to surgery, and the airway was markedly narrowed (Fig. 3). The CT scan revealed no laryngeal edema. Combined with the postoperative pathology, it was concluded that rapid growth of a rare upper airway lymphoma was the cause of the postoperative dyspnea.
No evidence of lymphoma involving the bone marrow was detected by bone marrow aspiration. A positron emission tomography (PET)/CT examination demonstrated that the left thyroid gland was diffusely enlarged and the glucose metabolism of the intratracheal soft tissues was high, raising the suspicion of lymphoma infiltration. No lymphoma lesions were identified in other areas of the body. On the 17th day after thyroidectomy, the patient received chemotherapy with the CHOP regimen combined with rituximab (R) 8 times; the specific regimen was as follows: $\mathrm{R} 800 \mathrm{mg}+$ vindesine $4 \mathrm{mg}$ $\mathrm{d} 1+$ cyclophosphamide $1.5 \mathrm{~g} \mathrm{~d} 1+$ epirubicin $120 \mathrm{mg} \mathrm{d} 1+$ prednisone $50 \mathrm{mg}$ b.i.d. d1-5 and mesna $0.4 \mathrm{~g}$. When chemotherapy was concluded, we attempted to plug the tube, and the patient experienced no dyspnea. At 6 months after the thyroidectomy, a tracheal stoma suture was performed, and dyspnea did not reoccur. After 8 rounds of chemotherapy, PET/CT re-examination revealed that the residual thyroid glucose metabolism was relatively decreased, and no abnormalities 

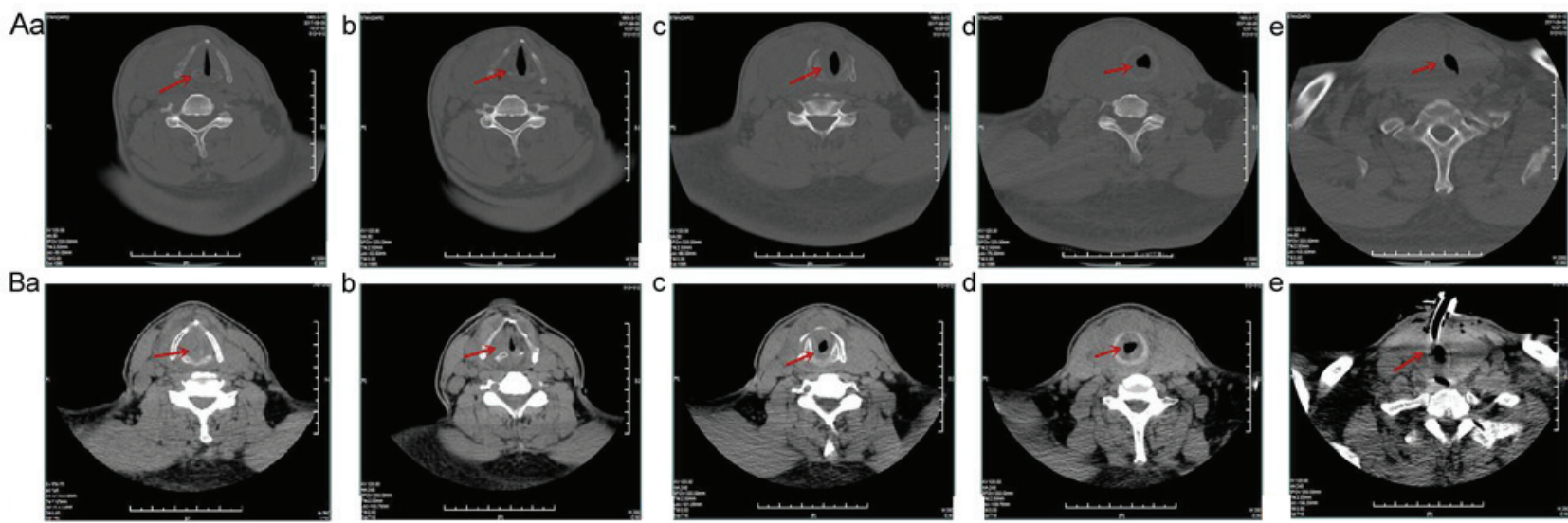

Figure 3. CT imaging examination of the intratracheal lymphoma. (A-a-e) Preoperative cervical CT images from the glottis to the fourth tracheal cartilage; (A-d): The arc right-side endotracheal soft tissue is a slightly thickened at the level of the third tracheal cartilage. (B-a-e) Postoperative cervical CT images from the glottis to the fourth tracheal cartilage: (B-a) Intratracheal soft tissue completely blocking the subglottic airway; (B-b-d) airway notably narrowed by thickened intratracheal soft tissue compared with A b-d, respectively; and (B-e) a tube was inserted into tracheal cavity by tracheotomy. Red arrows indicate the tracheal cavity. CT, computed tomography.

were detected in the trachea. On June 3, 2019, 21 months after thyroidectomy, no lymphoma recurrence in the patient was discovered by cervical CT or B-ultrasound.

\section{Discussion}

Dyspnea is one of the complications of thyroid surgery that may have severe consequences, including death. Common causes, including hematoma compression, tracheal collapse, laryngeal edema, and bilateral recurrent laryngeal nerve injury, are not to be overlooked, so that dyspnea can be prevented and/or treated it in a timely manner $(1,2)$. However, rarer causes may delay the etiological diagnosis, leading to a poor prognosis. To improve the prognosis of such patients, we herein report a case of dyspnea arising from a rare cause, namely the rapid growth of an intratracheal lymphoma after thyroid surgery.

The patient developed severe dyspnea 2 weeks after surgery and underwent a tracheostomy for temporary relief. Identifying the cause of postoperative dyspnea is crucial. The characteristics of the present case may be summarized as follows: i) Dyspnea was relieved after the right thyroidectomy, but worsened to the point of asphyxia 2 weeks later. ii) Dyspnea was relieved immediately after tracheotomy. iii) Preoperative imaging examination revealed a soft tissue shadow on the right side of the trachea. After the surgery, the soft tissue located in the subglottic cavity and upper trachea was notably thicker compared with prior to surgery, and the airway was significantly narrowed. iv) Postoperative pathology suggested thyroid non-Hodgkin B-cell lymphoma. v) After chemotherapy, from the completion of the tracheostomy to date, dyspnea did not reoccur, and no abnormalities were observed on PET/CT. i and ii) Consideration of upper airway obstruction; i-iii) consideration of upper airway obstruction caused by neoplasm in the trachea; $i-v)$ consideration of a rapid growth of rare upper airway lymphoma causing postoperative dyspnea after thyroidectomy.

First, reviewing the process of diagnosis and treatment, dyspnea occurred within 2 weeks rather than 48 h of surgery, no tracheomalacia was found intraoperatively, and postopera- tive CT did not reveal laryngeal edema; therefore, laryngeal edema and tracheal collapse can be excluded as the causes of dyspnea (3). No apparent hoarseness was observed in the patient, therefore excluding the possibility of bilateral recurrent laryngeal nerve injury. In addition, when postoperative dyspnea occurred, neck ultrasound examination excluded the possibility of hematoma compression. Thus, excluding other common causes of postoperative dyspnea, in combination with the rapid growth of the intratracheal tumor within 2 weeks postoperatively, its sensitivity to chemotherapy and its characteristic appearance on PET/CT imaging, even without a soft tissue biopsy, it was concluded clinically that the rapid growth of the intratracheal lymphoma was the cause of progressive dyspnea after thyroidectomy.

The patient was admitted to the hospital for dyspnea, and the etiological diagnosis was compression of the trachea by a thyroid mass based on medical history, physical and imaging examinations. Dyspnea was immediately relieved after right thyroidectomy, which suggested that compression of the trachea by a large mass on the right lobe of the thyroid gland was at least one of the causes of preoperative dyspnea. Combined with a rapid postoperative tumor growth in the trachea and preoperative CT indicating curved intratracheal soft tissue, intratracheal lymphoma was also determined to be one of the causes of preoperative dyspnea. Thus, when preoperative dyspnea occurs and CT indicates the presence of intratracheal soft tissue, a transbronchial biopsy should be performed to define the nature of the intratracheal soft tissue. If postoperative dyspnea occurs, the possibility of rapid growth of intratracheal soft tissues should also be considered.

Intratracheal tumor growth is a rare cause of airway stenosis. In addition, primary thyroid non-Hodgkin lymphoma is even rarer (4), and its clinical and imaging manifestations lack specificity; therefore, it is easy to misdiagnose and underdiagnose. Definitive diagnosis mainly relies on pathological examination, including immunohistochemistry $\left(\mathrm{CD} 20^{+}\right)(5,6)$. The chief complaint of patients is rapid enlargement of the mass, which may also invade the trachea. The patient in the present case was discharged from the hospital postoperatively 
following relief of the dyspnea; however, asphyxia developed rapidly within 2 weeks. Thyroid cancer invading the trachea may also cause dyspnea, and should be included in the differential diagnosis. The incidence of differentiated thyroid cancer invading the larynx and trachea is low, $13 \%$ (7). Causes of dyspnea in differentiated thyroid cancer cases include the primary tumor or paratracheal lymph node metastases invading the tracheal adventitia, or the tumor invading the whole thickness of the tracheal wall, finally leading to tracheal compression and stenosis. As differentiated thyroid carcinoma develops slowly and is characterized by a long disease course, the development of dyspnea is progressive, which differs significantly from undifferentiated carcinoma. The degree of malignancy of undifferentiated carcinoma is very high, and readily invades surrounding tissues and organs. If there is invasion of the trachea, the tumor will increase rapidly and result in dyspnea. However, undifferentiated carcinoma is relatively easy to cure and common among elderly female patients, with fast progression (8). Upon seeking medical advice, the majority of the patients have non-specific symptoms related to varying degrees of dysphagia, dyspnea, hoarseness or neck pain, and approximately $1 / 3$ of the patients have involvement of adjacent regional lymph nodes at initial presentation. Distant metastases also occur frequently; the most common site of metastasis is the lung, followed by the bone (9). Most undifferentiated carcinomas involving the trachea completely destroy the tracheal wall. Combined with the $\mathrm{CT}$ findings, the probability of an anaplastic thyroid carcinoma, which can be differentiated by biopsy, was low in our patient.

In conclusion, if common causes are excluded when postoperative dyspnea occurs, rare causes, such as the possibility of rapid growth of an intratracheal lymphoma, should be considered. Furthermore, preoperative needle biopsy of the thyroid mass should be routinely performed. If the patient is diagnosed with lymphoma following needle biopsy, chemotherapy may be immediately used rather than surgery.

\section{Acknowledgements}

Not applicable.

\section{Funding}

No funding was received.

\section{Availability of materials and data}

All the data generated and/or analyzed in the present study are included in the published manuscript.

\section{Authors' contributions}

All the authors have read and approved the final version of the manuscript for publication. CW drafted and wrote the manuscript, DD, GQ, XM collected the data, JW supported in the pathology, MZ gave suggestions, YJ did the thyroidectomy and gave suggestions, JL conceived the study and contributed to writing the manuscript.

\section{Ethics approval and consent to participate}

Not applicable.

\section{Patient consent for publication}

The patient agreed of the publication of this case details and any associated image with informed consent.

\section{Competing interests}

The authors declare that they have no competing interests.

\section{References}

1. Haugen BR, Alexander EK, Bible KC, Doherty GM Mandel SJ, Nikiforov YE, Pacini F, Randolph GW, Sawka AM, Schlumberger M, et al: 2015 American thyroid association management guidelines for adult patients with thyroid nodules and differentiated thyroid cancer: The American thyroid association guidelines task force on thyroid nodules and differentiated thyroid cancer. Thyroid 26: 1-133, 2016.

2. Rosato L, Avenia N, Bernante P, De Palma M, Gulino G, Nasi PG, Pelizzo MR and Pezzullo L: Complications of thyroid surgery: Analysis of a multicentric study on 14,934 patients operated on in Italy over 5 years. World J Surg 28: 271-276, 2004.

3. Goldfarb M, Perry Z, A Hodin R and Parangi S: Medical and surgical risks in thyroid surgery: Lessons from the NSQIP. Ann Surg Oncol 18: 3551-3558, 2011.

4. Green LD, Mack L and Pasieka JL: Anaplastic thyroid cancer and primary thyroid lymphoma: A review of these rare thyroid malignancies. J Surg Oncol 94: 725-736, 2006.

5. Stein SA and Wartofsky L: Primary thyroid lymphoma: A clinical review. J Clin Endocrinol Metab 98: 3131-3138, 2013.

6. Chen C, Yang Y, Jin L, Dong L, Zhang X and Xiang Y: Primary thyroid T-lymphoblastic lymphoma: A case report and review of the literature. Int J Clin Exp Pathol 7: 443-450, 2013.

7. Shindo ML, Caruana SM, Kandil E, McCaffrey JC, Orloff LA, Porterfield JR, Shaha A, Shin J, Terris D and Randolph G: Management of invasive well-differentiated thyroid cancer: An American head and neck society consensus statement. AHNS consensus statement. Head Neck 36: 1379-1390, 2014.

8. Sugitani I, Miyauchi A, Sugino K, Okamoto T, Yoshida A and Suzuki S: Prognostic factors and treatment outcomes for anaplastic thyroid carcinoma: ATC research consortium of Japan cohort study of 677 patients. World J Surg 36: 1247-1254, 2012.

9. Besic N and Gazic B: Sites of metastases of anaplastic thyroid carcinoma: Autopsy findings in 45 cases from a single institution. Thyroid 23: 709-713, 2013. 